\title{
Concept for a modular flight test camera system
}

\author{
Arne Nowak', Dr. Michael Schöberl', Ingo Bebermeier ${ }^{2}$ \\ ${ }^{1}$ Fraunhofer Institute for Integrated Circuits, Am Wolfsmantel 33, 91058 Erlangen, Germany, \\ arne.nowak@iis.fraunhofer.de, michael.schoeberl@iis.fraunhofer.de \\ 2 SEKAI Europe GmbH, Rissener Str. 102, 22880 Wedel, Germany, \\ ingo.bebermeier@sekai-europe.com
}

\begin{abstract}
:
Cameras have been widely in use for in-flight testing and other industrial applications to make observations and record them for later analysis for several years now. With the advent of cheap consumer action cameras like GoPro, this type of cameras became a viable option. However, consumer cameras do not cater for professional needs with regards to functionality, handling and other aspects. The Fraunhofer Institute for Integrated Circuits IIS and SEKAI Europe are working jointly on the development of a modular camera systems that consists of a front-end part with the lens and image sensor and a backend for image processing, control, video encoding and recording as well as streaming over a network interface. The modular approach makes it possible to provide different front-end modules that can be chosen depending on operational needs. One module will be very small and light-weight with an integrated fixed focal length lens while another module will be slightly larger but with a higher quality exchangeable lens and a larger imager chip. The backend can be freely configured regarding image processing and video encoding parameters. New functions can be added in the future by a software upgrade. Fraunhofer IIS and SEKAI Europe would like to present the concept to the audience and gather opinions for further improvement. Fraunhofer IIS and SEKAI Europe would like to present the concept at the etc 2016 (European Telemetry and Test Conference) to the audience and gather opinions for further improvement.
\end{abstract}

Key words: camera, video, recording, flight test

\section{Introduction}

Cameras are a very important tool in flight testing of airplanes and helicopters. They are used to make observation of moving parts of the aircraft in flight and to record them for thorough analysis on the ground after the test flight has been completed or to downlink compressed streams during the flight mission for immediate interaction. Modern digital camera systems offer a plethora of possibilities for this application with different types of image sensors, image postprocessing methods and video data compression algorithms. There are many small and light-weight cameras available but most of them do not offer the professional features that are required in flight test applications.

This paper gives an overview on the state of the art and the general requirements and use cases for flight test cameras. Afterwards the general concept of a modular flight test camera system is introduced and the two major components, camera heads and central processing unit, are described in detail. The paper concludes with an outlook on the further possibilities of this approach.

\section{Requirements from the industry and use cases}

In flight test applications there are diverse requirements for cameras depending on the actual use case. Where space for installation is limited the size of the camera and also often its weight play an important role. This also simplifies the temporary mounting that is carried out for each flight. For observing fast moving parts a high frame rate and global shutter image sensor is needed. In some situations it is important to simultaneously observe the inside and outside of the aircraft, for example in the cockpit. In these cases, both the flight situation through the window as well as the instrument panel and the pilot's actions should be visible. Here, a very high intra-frame dynamic range is necessary. For low light situations excellent sensitivity and low image noise are needed. Additionally, the camera system has to be able to cope with adverse environmental conditions: Very high and very low temperatures, low atmospheric pressure, vibration, possibly unstable power supply have to be taken into account. Last but not least the camera system has to provide appropriate electrical and logical interfaces for integration into the flight test and data recording 
environment and support methods for synchronization and timestamping of the image data to the data from other sensors. There also has to be a solution for later analysis of the image data in conjunction with other collected data often called meta-data.

\section{State of the art}

Several years ago, flight test applications where accomplished using analog cameras whose video signals were fed into appropriate, often digital, recording systems. While providing a very simple and universal interface the image quality of these cameras regarding spatial and temporal resolution is very limited. Sometimes, analog cameras are still used today like so-called lipstick cameras that have a very compact camera head [1].

The advent of digital cameras made it possible to more easily make use of higher spatial resolutions and higher frame rates. There are some solutions available especially developed for flight test purposes. Also, consumer grade cameras that became known as action cams are often used [2][3]. While specially developed cameras usually integrate well with the flight test environment this is difficult with consumer grade action cams that do not provide the ruggedness and interfaces necessary for this application. Common industrial digital cameras today use Ethernet as interface to a data recorder or store the video on memory cards. While action cams are often small, they lack professional possibilities for remote control. Furthermore, they mostly employ rolling shutter image sensors that lead to image distortion when observing moving objects. Lenses usually cannot be changed and are often very wide-angled which leads to geometric distortion of the image. Professional cameras are often bigger and heavier than action cams, which makes the temporary installation much more difficult.

Mobile phones and smartphones today employ high-quality camera modules for still images and video. The development and advances in this area have lead to a demise in sales of conventional compact digital cameras because the quality of mobile phone cameras has reached a comparable level [4]. These consumer system are able to deliver an excellent image quality that also sets an expectation and a reference for visual inspection systems. The employed integrated modules are very compact and consist of an image sensor chip with attached lenses. Concurrently, cameras used in industrial applications and machine vision also shrunk in size over the last few years.

For storage and transmission of the video data most solutions use the H.264 compressed video codec that provides a well-proven combination of video quality and low data rates. Hardware and software solutions for compression and decompression are available and make the use of the created files very easy.

\section{A modular concept for a flight test camera system}

We propose a modular concept for a flight test camera system that consists of a camera head module and a processing unit. The camera head contains an image sensor and the optics and is built as small and lightweight as possible. The processing unit contains the electronics which is necessary to control the camera head, process the images and create a compressed video stream. Both modules are connected by a costeffective and light-weight cable that provides power and control to the camera head and transmits the raw video image data signal from the camera head to the processing unit.

\section{Camera heads}

The concept includes the possibility to connect different types of camera heads to the processing unit. According to the different requirements of different use cases, each camera head model can be designed for either minimal size and weight, best dynamic range and sensitivity or maximum spatial resolution or frame rate. In order to achieve these goals the different models make use of different image sensor chips and lenses. Typical dimensions of a camera head are $2,5 \mathrm{~cm} \times 2,5 \mathrm{~cm} \times 4 \mathrm{~cm}$ (see Fig. 1, Fig. 2). For connecting the camera head to the central processing unit, all heads will use the same cabling and interface. This enables seamless exchange and extensions of the system. The use of cost-effective and lightweight cables with appropriate connectors makes is possible to install the cable in an aircraft and only attach the camera heads when needed for a test flight.

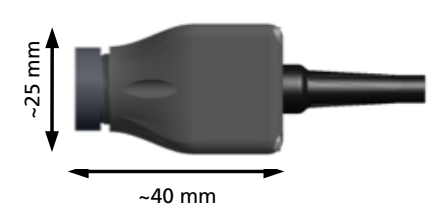

Fig. 1. Rendering of a camera head module 


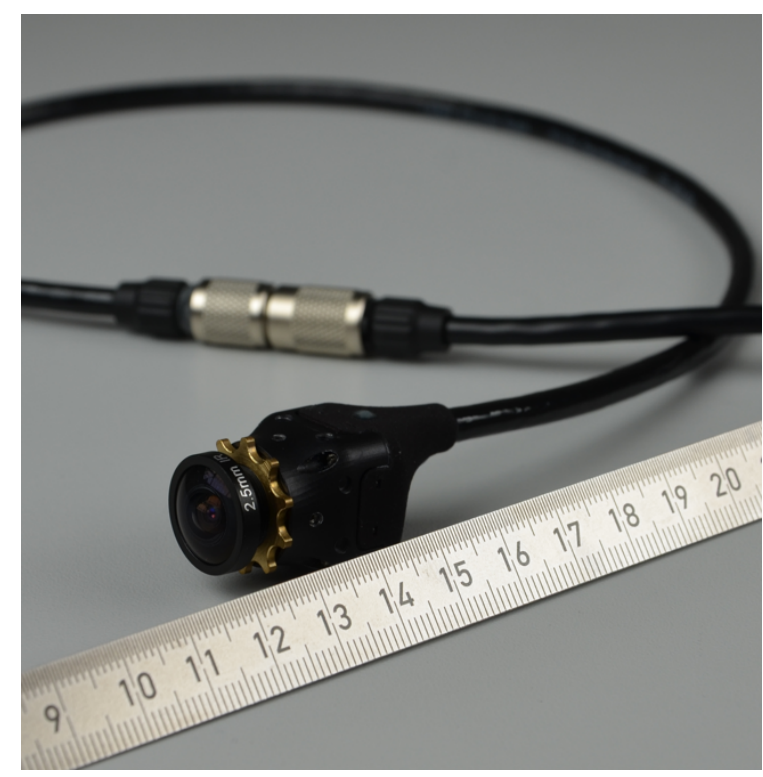

Fig. 2. Camera head and cable of a solution for digital media production

\section{Central processing unit}

The central processing unit is typically installed inside the aircraft together with other flight test equipment. Connection to the flight test data recorder or telemetry system is achieved via Ethernet which is used to control the camera system as well as to transmit the compressed video data stream. For video compression the H.264 video coding standard is used with the possibility to output one or more video streams at different data rates. This makes it possible to record a high quality video signal onboard the aircraft and simultaneously transmit a lower data rate video stream to a station on the ground via a telemetry system. By using H.264, the compressed video data stream is compatible with various standard video processing tools, which enables easy viewing, editing and archiving of the recorded video. The processing unit automatically recognizes which type of camera head is connected and adjusts the required image processing automatically.

\section{Outlook}

The modular approach of a flight test camera system as described in this paper makes it possible to use a specific camera head which provides the best suited features for that specific application or mission. Additionally, this approach provides the possibility to upgrade the system through the development of new camera heads when improved image sensors become available. Furthermore, it is possible to upgrade the processing unit in regards to hardware and software as well. A new hardware design can provide better performance while using existing camera heads. The functionality of existing camera systems can be improved via firmware upgrades of the processing unit.

The described modular system has a high potential to be deployed also in areas other than flight testing. Additional use cases can be e.g. found in video surveillance, digital media production, general testing applications and many more.

\section{References}

[1] http://www.toshiba.ch/ics/cp/products/micro cams/ik m51h/detail.html

[2] https://gopro.com/

[3] http://www.sony.com/electronics/actioncam/t/action-cam

[4] P. Cambou, JL. Jaffard, Status of the CMOS Image Sensor Industry report, Yole Développement, January 2015 